Supporting Information

\title{
Rapid Prediction of Chemical Ecotoxicity Through Genetic Algorithm Optimized Neural Network Models
}

Ping Hou ${ }^{1,2}, \mathrm{Bu}$ Zhao ${ }^{1,2}$, Olivier Jolliet ${ }^{3}$, Ji Zhu ${ }^{4}$, Peng Wang ${ }^{5}$, and Ming $\mathrm{Xu}^{1,7^{*}}$

${ }^{1}$ School for Environment and Sustainability, University of Michigan, 440 Church St., Ann Arbor, MI, 48109, USA.

${ }^{2}$ Michigan Institute for Computational Discovery \& Engineering, University of Michigan, 500 Church St., Ann Arbor, MI, 48109, USA.

${ }^{3}$ Environmental Health Sciences, School of Public Heath, University of Michigan, 1415

Washington Heights, Ann Arbor, MI, 48109, USA.

${ }^{4}$ Department of Statistics, University of Michigan, 1085 South University, Ann Arbor, MI, 48109, USA.

${ }^{5}$ Department of Electrical and Computer Engineering, University of Kentucky, 512 Administration Drive, Lexington, KY, 40506, USA.

${ }^{6}$ Department of Mechanical Engineering, University of Kentucky, 151 Ralph G. Anderson Building, Lexington, KY, 40506, USA.

${ }^{7}$ Department of Civil and Environmental Engineering, University of Michigan, 2350 Hayward Street, Ann Arbor, MI, 48109, USA.

*Correspondence to: mingxu@umich.edu.

This Supporting Information includes 3 pages, 2 figures and 1 table. 
Table S1. Commonly used activation functions.

\begin{tabular}{|c|c|c|}
\hline Name & Input output relation & $\begin{array}{l}\text { Graphic } \\
\text { representation }\end{array}$ \\
\hline $\begin{array}{l}\text { ReLU (Rectified Linear } \\
\text { Unit) }\end{array}$ & $\begin{array}{c}f(x)=\alpha *(x-\text { threshold }) \quad x<\text { threshold } \\
f(x)=x \text { threshold } \leq x \leq \text { max_value } \\
f(x)=\text { max_value } x \geq \text { max_value }\end{array}$ & \\
\hline $\begin{array}{l}\text { ELU (Exponential linear } \\
\text { unit) }\end{array}$ & $\begin{array}{c}f(x)=\alpha *(\exp (x)-1) \quad x<0 \\
f(x)=x \quad x \geq 0\end{array}$ & \\
\hline $\begin{array}{l}\text { TanH (Hyperbolic } \\
\text { tangent) }\end{array}$ & $f(x)=\frac{e^{x}-e^{-x}}{e^{x}+e^{-x}}$ & \\
\hline Sigmoid & $f(x)=\frac{1}{1+e^{-x}}$ & \\
\hline Hard_sigmoid & $\begin{array}{c}f(x)=0 \quad x<-2.5 \\
f(x)=0.2 * x+0.5 \quad-2.5 \leq x \leq 2.5 \\
f(x)=1 \quad x \geq 2.5\end{array}$ & \\
\hline SoftPlus & $f(x)=\log (\exp (x)+1)$ & \\
\hline Linear & $f(x)=x$ & \\
\hline
\end{tabular}




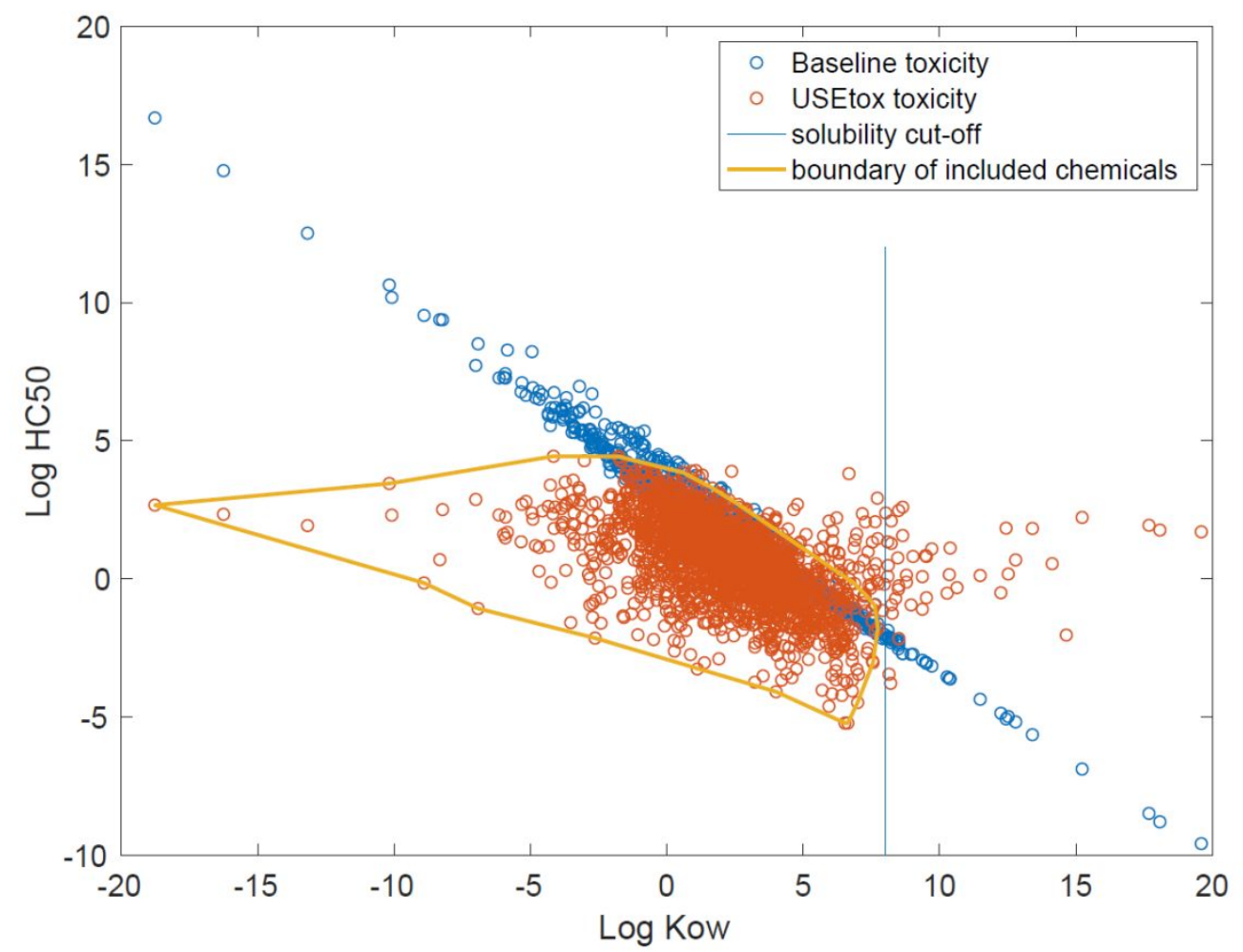

Figure S1. Data filtering result
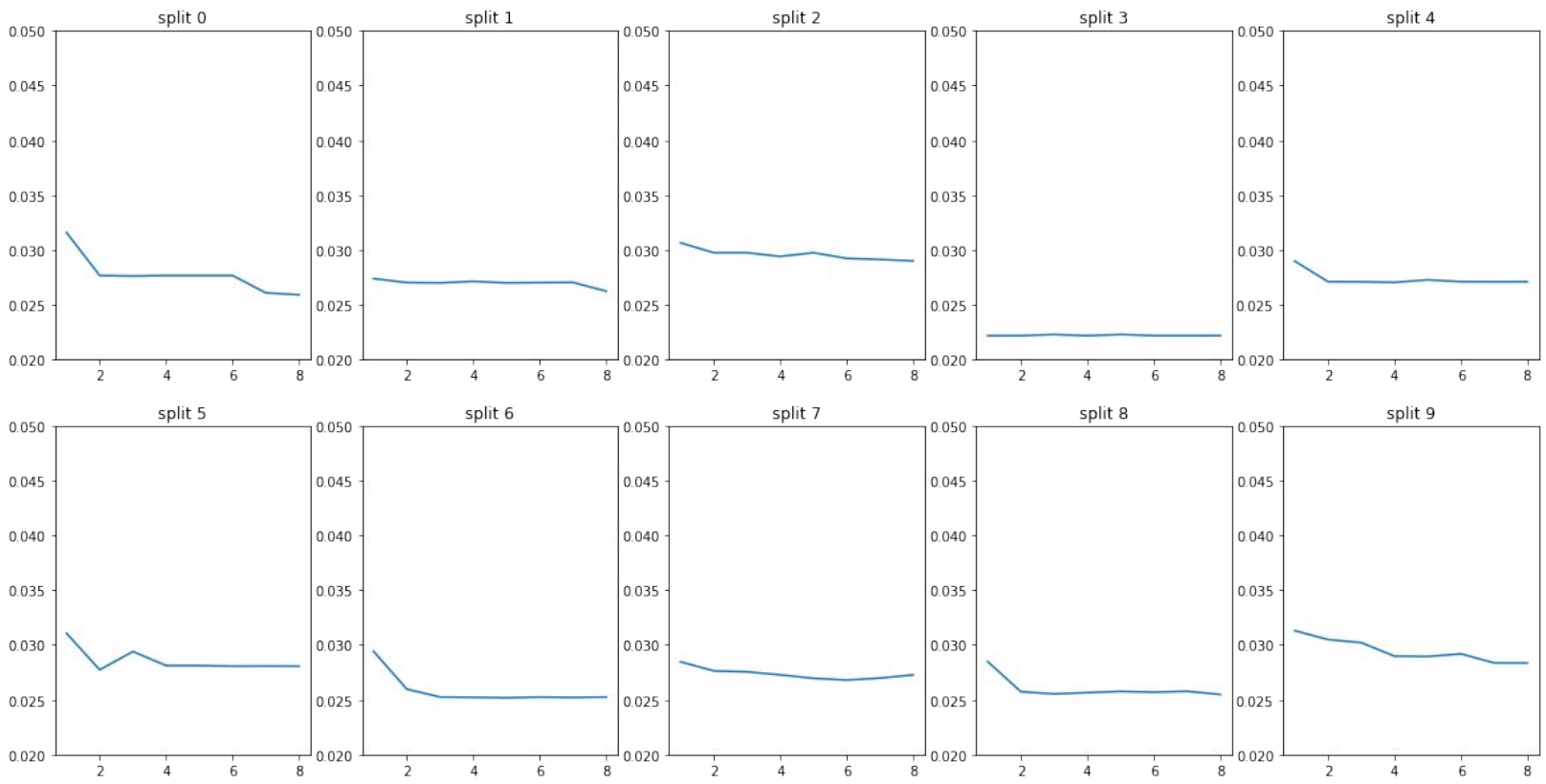

Figure S2. Fitness (i.e., validation MSE) of the best model along eight generations. 